\title{
Anisotropic hydrodynamics and early stages of heavy-ion collisions
}

\author{
Wojciech Florkowski ${ }^{1,2, a}$ \\ ${ }^{1}$ Institute of Physics, Jan Kochanowski University, PL-25406 Kielce, Poland \\ ${ }^{2}$ The H. Niewodniczański Institute of Nuclear Physics, Polish Academy of Sciences, \\ PL-31342 Kraków, Poland
}

\begin{abstract}
New formulations of relativistic dissipative hydrodynamics are discussed in the context of possible applications to describe early stages of relativistic heavy-ion collisions. The efficacy of different approaches is tested by making comparisons with the exact solutions of the kinetic equation.
\end{abstract}

\section{Introduction}

Relativistic viscous hydrodynamics [1-13] is the main building block in the standard model of relativistic heavyion collisions, which includes three important parts: the modeling of initial conditions, the application of relativistic hydrodynamics to describe the space-time evolution of matter, and, finally, the modeling of the freeze-out conditions. The inclusion of viscous effects is important as it improves the overall description of the data (as compared to earlier perfect-fluid applications) and makes the applied theory consistent with general physics arguments that the fluid viscosity cannot be zero $[14,15]$.

Probably, the weakest point in the scheme outlined above is the transition from the initial state, which is very well described within the CGC theory or the Glauber model, to the hydrodynamic stage where one uses the concept of local thermalisation. The standard viscous hydrodynamics relies on the expansion around the local equilibrium state. This expansion may be questioned at the very early stages of relativistic heavy-ion collisions where the space-time gradients are large and the viscous corrections become very substantial in spite of the fact that the ratio of the shear viscosity to entropy density is minimal, $\eta / s \sim 1 /(4 \pi)[15]$.

The results of microscopic models suggest that the initial longitudinal pressure is reduced to zero (or may take even negative values due to the presence of the longitudinal colour fields). Consequently, the viscous corrections at the very early stages of the collisions should be of the same order as the leading (equilibrium) term. This leads to several difficulties one may encounter in the application of the standard dissipative hydrodynamics [16]. Such problems initiated a new approach which is now referred to as anisotropic hydrodynamics (aHydro). The latter is based on the expansion in which large momentumspace anisotropies are built directly into the leading order [17-26]. The recent methods applied to improve the

\footnotetext{
ae-mail: Wojciech.Florkowski@ifj.edu.pl
}

efficacy of the more traditional hydrodynamic approaches include complete second-order treatments [27] and thirdorder treatments [28-30],

In this note we discuss the applications of standard viscous hydrodynamics and anisotropic hydrodynamics in the context of the exact solutions of the Boltzmann kinetic equation [31-33]. We consider a one-dimensional boostinvariant expansion, which may be a good approximation for the early dynamics.

In our earlier works, we studied the effects connected with the shear viscosity and showed that recent formulations of second-order viscous hydrodynamics [27] agree better with the exact solutions of the kinetic equation than the standard Israel-Stewart approach [1, 2]. Here, we concentrate in more detail on the effects connected with the bulk viscosity [34]. It has been argued that the finite bulk viscosity coefficient leads to improved description of the flow harmonics in ultracentral collisions [35]. On the theory side, it has been demonstrated that the correct description of the bulk viscous pressure demands the correct treatment of the bulk-viscous coupling $[36,37]$.

\section{Exact solutions of Boltzmann equation}

Having in mind very early dynamics of the central rapidity region of heavy-ion collisions, we may assume that the produced system is boost-invariant and azimuthally symmetric. In this case, one can use a simple form of the kinetic equation [31-33]

$$
\frac{\partial f}{\partial \tau}=\frac{f^{\mathrm{eq}}-f}{\tau_{\mathrm{eq}}} .
$$

Here $f(x, p)$ is the phase-space distribution function, $\tau=$ $\sqrt{t^{2}-z^{2}}$ is the longitudinal proper time, and $\tau_{\text {eq }}$ is the relaxation time. The condition of boost invariance implies that $f(x, p)$ may depend only on: $\tau, w$ and $p_{T}$. The boostinvariant variable $w$ is defined by the formula $w=t p_{L}-z E$, where $p_{L}\left(p_{T}\right)$ is the longitudinal (transverse) momentum 

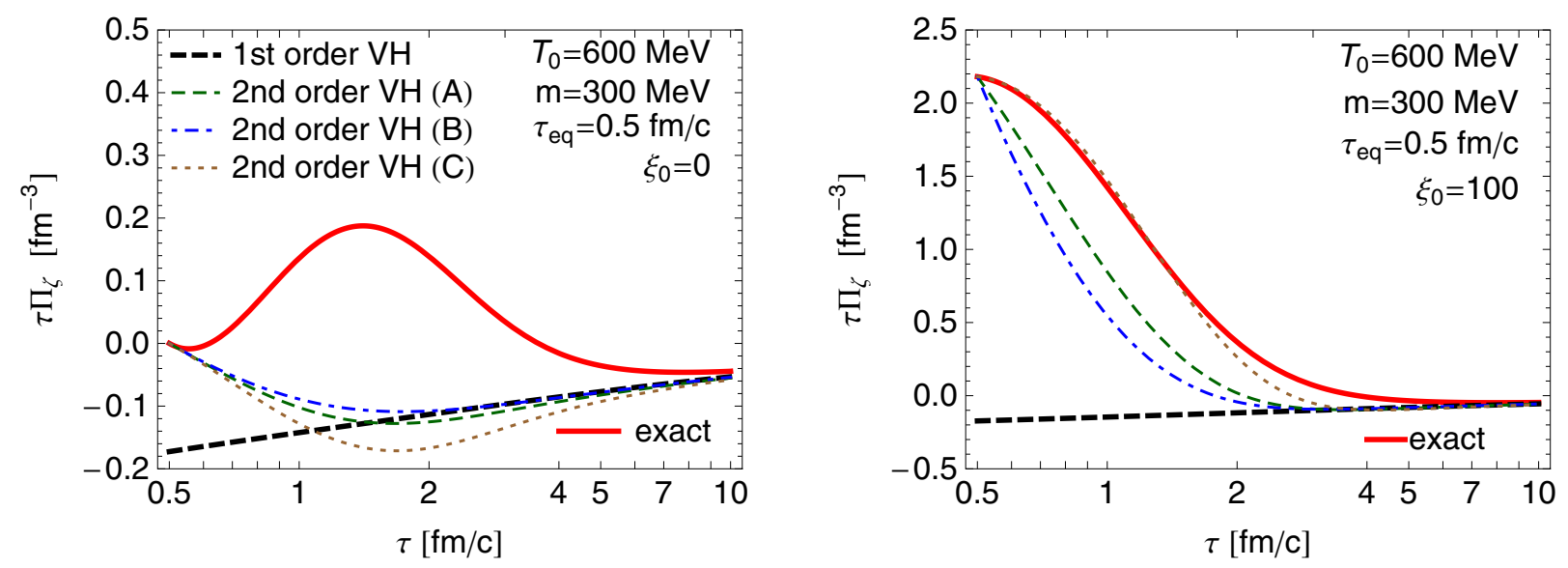

Figure 1. Time dependence of the bulk viscous pressure (multiplied by the proper time) obtained from the kinetic theory and various hydrodynamic equations (figure taken from [33]).

of a particle and $E$ is its energy. The equilibrium background distribution function $f^{\text {eq }}$ appearing in Eq. (1) takes the form

$$
f^{\mathrm{eq}}\left(\tau, w, p_{T}\right)=\frac{2}{(2 \pi)^{3}} \exp \left[-\frac{\sqrt{w^{2}+p_{T}^{2} \tau^{2}}}{T(\tau) \tau}\right],
$$

where $T$ is an effective temperature. The latter is obtained from the Landau matching condition that the energy density obtained from the distribution $f$ is equal to the energy density obtained with the equilibrium distribution $f_{\text {eq }}$.

The first moment of the kinetic equation gives the energy-momentum tensor that should be conserved,

$$
T^{\mu v}(\tau)=g_{0} \int d P p^{\mu} p^{v} f\left(\tau, w, p_{T}\right), \quad \partial_{\mu} T^{\mu v}=0 .
$$

Using the symmetry properties of the distribution function, we rewrite (3) in the form $[17,22]$

$$
T^{\mu v}=\left(\mathcal{E}+\mathcal{P}_{T}\right) u^{\mu} u^{v}-\mathcal{P}_{T} g^{\mu v}+\left(\mathcal{P}_{L}-\mathcal{P}_{T}\right) z^{\mu} z^{v},
$$

where $u^{\mu}=(t, 0,0, z) / \tau$ and $z^{\mu}=(z, 0,0, t) / \tau$. The fourvector $z$ defines the beam direction. The energy density $\mathcal{E}$ and the two (longitudinal, $\mathcal{P}_{L}$, and transverse, $\mathcal{P}_{T}$ ) pressures are defined as the integrals over the distribution function $f$ multiplied by the appropriate combinations of the momentum. The factor $g_{0}$ in Eq. (3) describes internal degrees of freedom different than spin. The spin degeneracy is included explicitly in (2).

The initial condition at the time $\tau=\tau_{0}$ used to solve numerically Eq. (1) corresponds to the RomatschkeStrickland (RS) form of the distribution function [38]

$$
f_{0}\left(w, p_{T}\right)=\frac{2}{(2 \pi)^{3}} \exp \left[-\frac{\sqrt{\left(1+\xi_{0}\right) w^{2}+p_{T}^{2} \tau_{0}^{2}}}{\Lambda_{0} \tau_{0}}\right] .
$$

This form is reduced to an isotropic Boltzmann distribution if the anisotropy parameter $\xi_{0}=\xi\left(\tau_{0}\right)$ is set equal to zero. In this case, the transverse momentum scale
$\Lambda_{0}=\Lambda\left(\tau_{0}\right)$ is equal to the system's initial temperature $T_{0}$. The methods of solving Eq. (1) were explained in more detail in [31-33].

The solution of the kinetic equation allows us to calculate the bulk viscous pressure from the formula

$$
\Pi_{\zeta}^{k}(\tau)=\frac{1}{3}\left[\mathcal{P}_{\|}(\tau)+2 \mathcal{P}_{\perp}(\tau)-3 \mathcal{P}_{\mathrm{eq}}(\tau)\right]
$$

where $\mathcal{P}_{\text {eq }}$ and $\mathcal{E}$ are connected through the equation of state (for the massive gas). In Fig. 1 the red solid curves show the time dependence of the bulk viscous pressure obtained from the kinetic equation for the two different initial conditions characterised by the momentum anisotropy parameter $\xi_{0}$. The initial temperature of the system in the two cases is $T_{0}=600 \mathrm{MeV}$, the effective particle mass is $m=300 \mathrm{MeV}$, and the equilibration time $\tau_{\mathrm{eq}}=0.5 \mathrm{fm}$. We also take $\tau_{0}=\tau_{\text {eq }}$.

\section{Bulk viscous pressure in dissipative and anisotropic hydrodynamics}

The most common applications of dissipative hydrodynamics use the following three equations to determine the time evolution of the bulk pressure:

$$
\begin{aligned}
\tau_{\Pi} \dot{\Pi}_{\zeta}+\Pi_{\zeta} & =-\frac{\zeta}{\tau}-\frac{1}{2} \tau_{\Pi} \Pi_{\zeta}\left[\frac{1}{\tau}-\left(\frac{\dot{\zeta}}{\zeta}+\frac{\dot{T}}{T}\right)\right], \\
\tau_{\Pi} \dot{\Pi}_{\zeta}+\Pi_{\zeta} & =-\frac{\zeta}{\tau}-\frac{4}{3} \tau_{\Pi} \Pi_{\zeta} \frac{1}{\tau}, \\
\tau_{\Pi} \dot{\Pi}_{\zeta}+\Pi_{\zeta} & =-\frac{\zeta}{\tau} .
\end{aligned}
$$

We have solved these equations with the initial conditions corresponding exactly to those used in the kinetic-theory approach described in the previous Section. The results are shown in Fig. 1, with the cases (A), (B), and (C) corresponding to Eqs. (7), (8), and (9), respectively. The thick dashed line shows the first-order result where the 


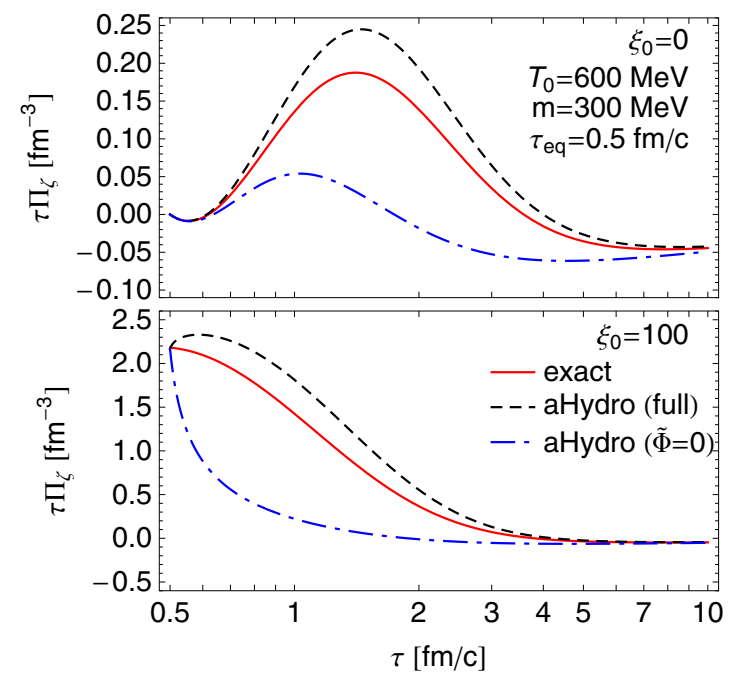

Figure 2. Time dependence of the bulk viscous pressure for two different initial anisotropy parameters: $\xi_{0}=0$ (upper panel) and $\xi_{0}=100$ (lower panel). The solid lines show the exact result. The dashed-dotted lines represent the predictions of aHydro formulated in Ref. [39], while the dashed lines represent the predictions of Ref. [40] (figure taken from [40]).

bulk pressure is directly expressed by the bulk viscosity, i.e., $\Pi_{\zeta}=-\zeta / \tau$. A surprising result of our comparisons is that none of the equations (7)-(9) reproduces correctly the numerical result.

In the anisotropic hydrodynamics approach introduced in Refs. [24, 39], one assumes that the leading-order distribution function takes always the RS form with the parameters $\xi$ and $\Lambda$ depending on the proper time $\tau$. The hydrodynamic equations are obtained from the first and second moments of the kinetic equation (1). In a more recent formulation [40], the leading-order phase-space distribution function depends on an extra parameter $\tilde{\Phi}$, and the dynamic equations follow from the zeroth, first and second moments of the kinetic equation (1) ${ }^{1}$. The results obtained with the two versions of aHydro are presented in Fig. 2 and compared with the results of the kinetic theory. One finds that the inclusion of an additional parameter in the ansatz for the distribution function improves the agreement with the kinetic theory results. One also finds that aHydro describes the evolution of the bulk pressure significantly better than the dissipative hydrodynamics based on Eqs. (7)(9). Discrepancies between the results of the kinetic theory and dissipative hydrodynamics may be connected with the absence of the shear-bulk coupling in Eqs. (7)-(9). This has been demonstrated recently in Refs. [36, 37].

\section{Conclusions}

Detailed comparisons between the exact results of the kinetic theory and the predictions of hydrodynamic models

\footnotetext{
${ }^{1}$ There exists a different formulation of aHydro, where some part of anisotropy is treated perturbatively by adding corrections to the leadingorder term, see Ref. [41]
}

allow us to choose the correct form of the hydrodynamic equations and kinetic coefficients. In this way, we select the appropriate structure of the hydrodynamic equations that may be used to model relativistic heavy-ion collisions. This procedure is especialy important in the case of the early stages of the collisions where large gradients require the use of more complete, higher-order approximations. Anisotropic hydrodynamics seems to be a very good alternative for these approaches.

In this note we have presented the analysis of onedimensional systems. Very recently, the exact solutions of the two-dimensional systems have become also available [42-44] (for the systems which are boost-invariant and azimuthally symmetric) but they are restricted to conformal systems. Therefore, they are suitable for studies of the effects connected with shear viscosity.

This work was supported in part by the Polish National Science Center with decision No. DEC2012/06/A/ST2/00390.

\section{References}

[1] W. Israel and J. M. Stewart, Annals Phys. 118, 341 (1979).

[2] A. Muronga, Phys. Rev. C 69, 034903 (2004).

[3] R. Baier, P. Romatschke and U. A. Wiedemann, Phys. Rev. C 73, 064903 (2006)

[4] P. Romatschke and U. Romatschke, Phys. Rev. Lett. 99, 172301 (2007).

[5] K. Dusling and D. Teaney, Phys. Rev. C 77, 034905 (2008).

[6] M. Luzum and P. Romatschke, Phys. Rev. C 78, 034915 (2008).

[7] H. Song and U. W. Heinz, J. Phys. G 36, 064033 (2009).

[8] G. S. Denicol, T. Kodama and T. Koide, J. Phys. G 37, 094040 (2010).

[9] B. Schenke, S. Jeon and C. Gale, Phys. Lett. B 702, 59 (2011).

[10] C. Shen, U. Heinz, P. Huovinen and H. Song, Phys. Rev. C 84, 044903 (2011).

[11] P. Bozek, Phys. Lett. B 699, 283 (2011).

[12] H. Niemi, G. S. Denicol, P. Huovinen, E. Molnar and D. H. Rischke, Phys. Rev. Lett. 106, 212302 (2011).

[13] P. Bozek and I. Wyskiel-Piekarska, Phys. Rev. C 85, 064915 (2012).

[14] P. Danielewicz and M. Gyulassy, Phys. Rev. D 31, 53 (1985).

[15] P. Kovtun, D. T. Son and A. O. Starinets, Phys. Rev. Lett. 94, 111601 (2005).

[16] M. Martinez and M. Strickland, Phys. Rev. C 79, 044903 (2009).

[17] W. Florkowski and R. Ryblewski, Phys. Rev. C 83, 034907 (2011).

[18] M. Martinez and M. Strickland, Nucl. Phys. A 848, 183 (2010).

[19] R. Ryblewski and W. Florkowski, J. Phys. G 38, 015104 (2011). 
[20] M. Martinez and M. Strickland, Nucl. Phys. A 856, 68 (2011).

[21] R. Ryblewski and W. Florkowski, Eur. Phys. J. C 71, 1761 (2011).

[22] M. Martinez, R. Ryblewski and M. Strickland, Phys. Rev. C 85, 064913 (2012).

[23] R. Ryblewski and W. Florkowski, Phys. Rev. C 85, 064901 (2012).

[24] W. Florkowski and R. Ryblewski, Phys. Rev. C 85, 044902 (2012).

[25] L. Tinti and W. Florkowski, Phys. Rev. C 89, 034907 (2014).

[26] L. Tinti, arXiv:1411.7268 [nucl-th].

[27] G. S. Denicol, H. Niemi, E. Molnar and D. H. Rischke, Phys. Rev. D 85, 114047 (2012).

[28] A. El, Z. Xu and C. Greiner, Phys. Rev. C 81, 041901 (2010).

[29] A. Jaiswal, Phys. Rev. C 88, 021903 (2013).

[30] C. Chattopadhyay, A. Jaiswal, S. Pal and R. Ryblewski, arXiv:1411.2363 [nucl-th].

[31] W. Florkowski, R. Ryblewski and M. Strickland, Nucl. Phys. A 916, 249 (2013).

[32] W. Florkowski, R. Ryblewski and M. Strickland, Phys. Rev. C 88, 024903 (2013).
[33] W. Florkowski, E. Maksymiuk, R. Ryblewski and M. Strickland, Phys. Rev. C 89, 054908 (2014).

[34] G. S. Denicol, S. Jeon and C. Gale, arXiv:1403.0962 [nucl-th].

[35] J. B. Rose, J. F. Paquet, G. S. Denicol, M. Luzum, B. Schenke, S. Jeon and C. Gale, arXiv:1408.0024 [nucl-th].

[36] G. S. Denicol, W. Florkowski, R. Ryblewski and M. Strickland, Phys. Rev. C 90, 044905 (2014).

[37] A. Jaiswal, R. Ryblewski and M. Strickland, Phys. Rev. C 90, 044908 (2014).

[38] P. Romatschke and M. Strickland, Phys. Rev. D 68, 036004 (2003).

[39] W. Florkowski, R. Ryblewski, M. Strickland and L. Tinti, Phys. Rev. C 89, 054909 (2014).

[40] M. Nopoush, R. Ryblewski and M. Strickland, Phys. Rev. C 90, 014908 (2014).

[41] D. Bazow, U. W. Heinz and M. Strickland, Phys. Rev. C 90, 044908 (2014).

[42] G. S. Denicol, U. W. Heinz, M. Martinez, J. Noronha and M. Strickland, Phys. Rev. Lett. 113, 202301 (2014).

[43] G. S. Denicol, U. W. Heinz, M. Martinez, J. Noronha and M. Strickland, arXiv:1408.7048 [hep-ph].

[44] M. Nopoush, R. Ryblewski and M. Strickland, arXiv:1410.6790 [nucl-th]. 